\title{
Worldwide experiences and opinions of healthcare providers on eHealth for patients with interstitial lung diseases in the COVID-19 era
}

\begin{abstract}
To the Editor:
Copyright @The authors 2021

This version is distributed under the terms of the Creative Commons Attribution NonCommercial Licence 4.0. For commercial reproduction rights and permissions contact permissions@ersnet.org

Received: 18 June 2021 Accepted: 21 June 2021

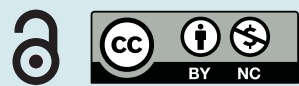

Interstitial lung diseases (ILD) comprise a group of pulmonary diseases characterised by diffuse parenchymal abnormalities, which can lead to irreversible pulmonary fibrosis [1].

In current practice, outpatient clinic visits including pulmonary function measurements every 3-6 months are typically used to monitor disease course and determine treatment strategies [2]. The availability of digital technologies may enhance efficiency and precision of disease monitoring at a lower burden to both patients and clinicians, and may change routine clinical practice.

The coronavirus disease 2019 (COVID-19) pandemic has impacted almost all aspects of care for patients with ILD. Decreased hospital capacity and avoidance of face-to-face contact have fostered an accelerated implementation of eHealth. Replacing outpatient clinic visits with video consultation and home monitoring of patients have been proposed as strategies to provide ongoing care for patients with ILD, given their increased risk for severe outcomes associated with COVID-19 infection [3-6]. However, the extent to which healthcare providers (HCPs) have implemented eHealth in daily practice, and their perspectives on digitalisation of care for patients with ILD have not yet been assessed. These insights will help further shape digital healthcare.

The aim of our study was to characterise the experience of HCPs around the world on eHealth tools for patients with ILD, and to understand opportunities and barriers for further implementation of eHealth and home monitoring in this patient population.

Between October 2020 and January 2021, HCPs with ILD expertise were invited by email to complete a 28 question online survey using Survio ${ }^{\circledR}$ (www.survio.com). HCPs provided informed consent for participation. eHealth was defined as "the use of technology to improve health and/or quality of healthcare", and online home monitoring as "tracking clinical results measured at home using an online application”. Microsoft Excel and SPSS statistics version.25.0.0.1 were used for data collection and analysis.

In total, 286 HCPs from 54 different countries responded: 89.2\% were pulmonologists, 7\% rheumatologists, $1.7 \%$ ILD specialist nurses, and $2.1 \%$ others. The mean \pm SD age was $46 \pm 9.9$ years, and $50 \%$ were male. $52.8 \%$ of HCPs had over 10 years of experience with care for patients with ILD, and 49.7\% reported seeing more than 100 patients with ILD per year.

62.2\% of HCPs stated that patients are able to communicate with the ILD clinic via email, and $36.0 \%$ reported that patients have the possibility to view their own clinical records online.

Approximately half of HCPs currently use eHealth; a minority (8.4\%) already started before the COVID-19 pandemic (figure 1a). Of the 157 HCPs with eHealth experience, 39.5\% use it in daily care, $25.5 \%$ for research purposes, and $35.0 \%$ for both research and daily care. All respondents from Oceania

\section{Shareable abstract (@ERSpublications)}

The \#COVID19 pandemic has led to an increase in the use of eHealth for patients with interstitial lung disease. Healthcare providers worldwide are positive towards further implementation of eHealth for research and clinical practice. https://bit.ly/3h2545M

Cite this article as: Nakshbandi G, Moor CC, Johannson KA, et al. Worldwide experiences and opinions of healthcare providers on eHealth for patients with interstitial lung diseases in the COVID-19 era. ERJ Open Res 2021; 7: 00405-2021 [DOI: 10.1183/23120541.00405-2021].
\end{abstract}


a)

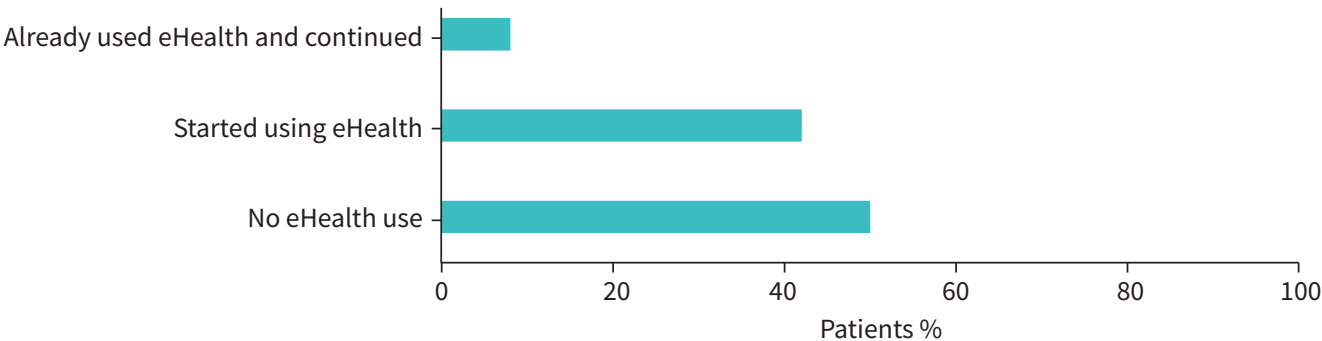

b)

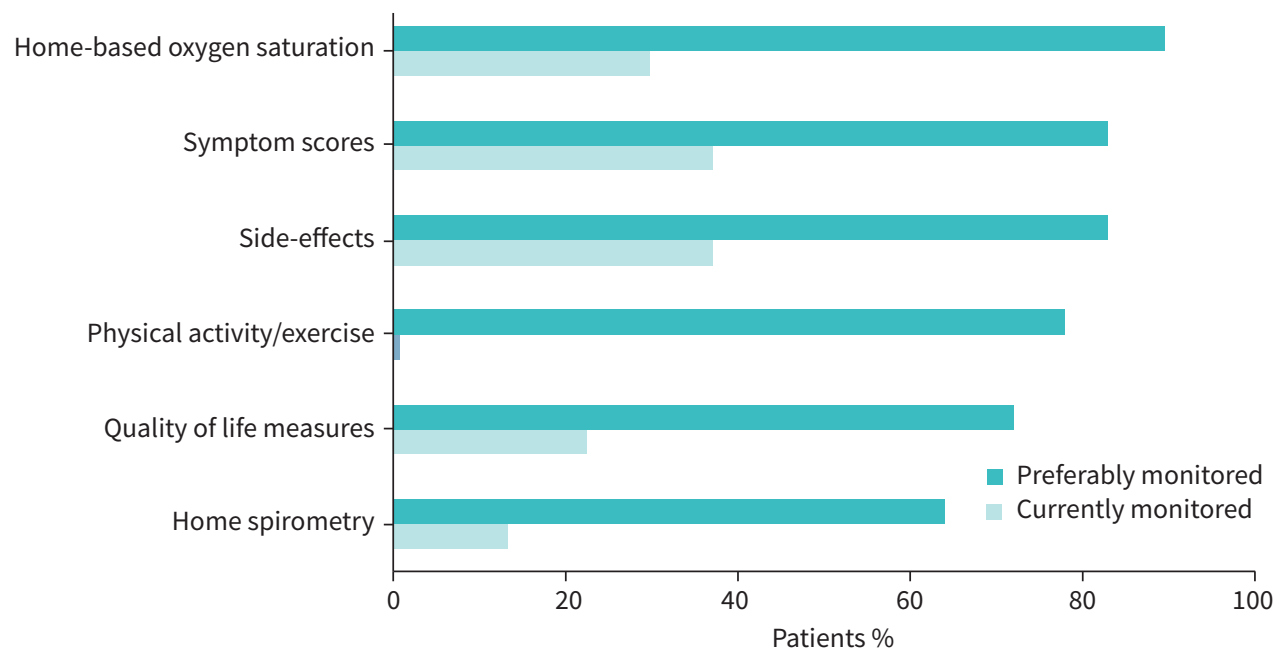

c)

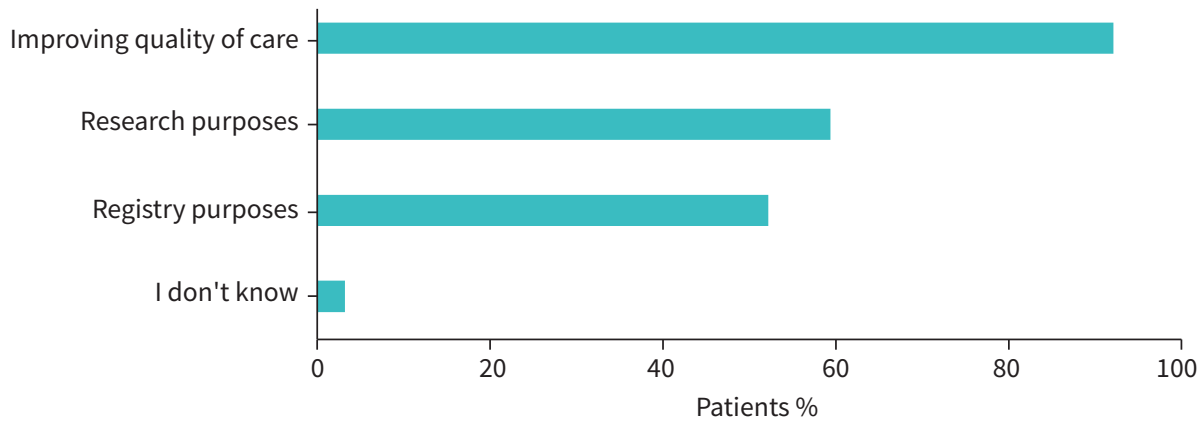

d)

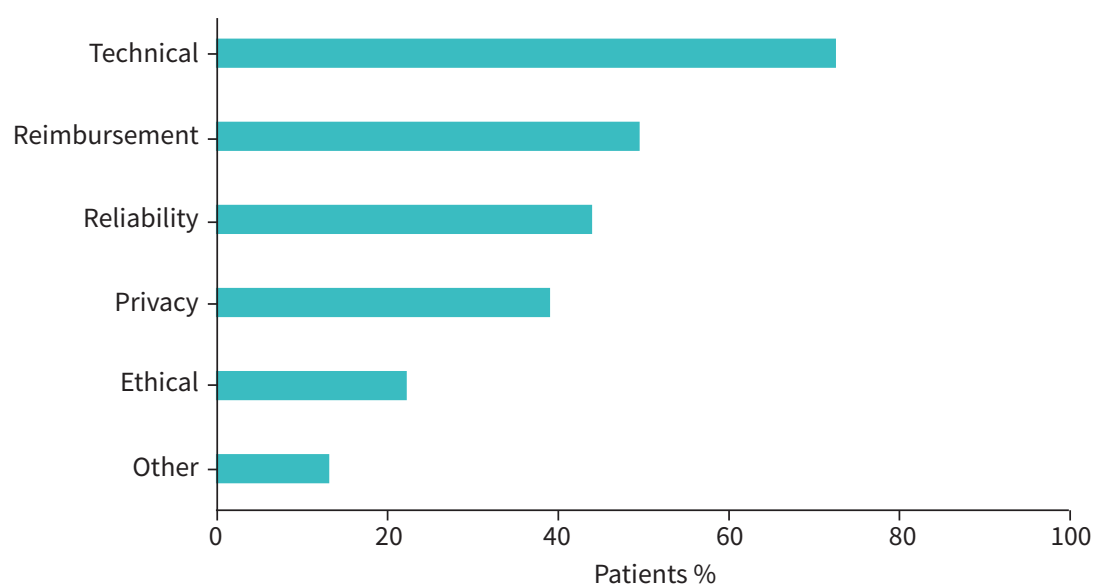

FIGURE 1 Worldwide experiences and opinions on eHealth for patients with interstitial lung disease. a) Effects of the coronavirus disease 2019 pandemic. b) Preferences and current use of home monitoring. c) Added value of online home monitoring. d) Hurdles for implementation. 
$(100.0 \%, n=6)$, and the majority from North America $(77.6 \%, n=58)$, and South America $(58.5 \%, n=41)$ used eHealth. In Europe, approximately half of participating HCPs $(47.7 \%, n=149)$ currently use eHealth, and in Asia around one-third (35.5\%, $\mathrm{n}=31)$. The only African HCP in this survey did not use eHealth. The most frequently used forms of eHealth are video consultations (67.6\%), online patient portals (30.2\%), online home monitoring (21.8\%), and online self-help applications (8.9\%). Of the 129 HCPs without experience, $41.9 \%$ state that they would like to use eHealth, but don't know how to set it up.

HCPs would like to monitor home-based oxygen saturation, symptom scores, side-effects, physical activity levels, quality of life, and home spirometry. However, only a minority are currently collecting these parameters (figure 1b). Few HCPs also mentioned interest in environmental exposures, treatment compliance, tele-rehabilitation, laboratory results, blood pressure, and heart rate as part of home monitoring.

Almost all HCPs (96.5\%) believe online home monitoring to be of added value, either for improving quality of care, research or registry purposes (figure 1c). If patients collect online data, $75.2 \%$ of HCPs would like to have access to the results; $18.9 \%$ would want patients to contact their HCP if their condition worsens. Only $1 \%$ think home monitoring data are of predominant interest for patients. Three-quarters of HCPs stated that they would like to receive automated alerts if data collected by patients show disease deterioration. Currently collected online data is mainly followed up by medical specialists, ILD specialist nurses, and research nurses. Most HCPs (80.8\%) would like to have contact with patients via video consultations.

Implementation of eHealth may also pose challenges. Technical issues were mentioned as the main challenge by most HCPs, followed by reimbursement, reliability, privacy, and ethical issues (figure 1d). Other mentioned hurdles for implementation were management and interpretation of large volumes of data, suitability of eHealth for some patients, accessibility, and time and human resources to monitor results. $59.8 \%$ of HCPs stated that they do not have staff available to teach patients how to use home monitoring applications. $34.3 \%$ stated that there is no reimbursement for eHealth in their country, and $37.8 \%$ do not know if there is reimbursement available.

Our results reveal that most HCPs were positive towards implementation of eHealth, including online home monitoring applications, not only for daily care, but also for research and registry purposes. Before the COVID-19 pandemic, $<10 \%$ of HCPs used eHealth. The COVID-19 pandemic has stimulated the implementation of eHealth for patients with ILD, with half of surveyed HCPs currently using a form of eHealth.

Most HCPs would like to use different home monitoring applications, such as home-based oxygen saturation, symptom scores, side-effect monitoring, physical activity trackers, quality of life measures, and home spirometry. Previous studies have shown that online home monitoring is feasible, reliable and can even be beneficial for patients with ILD [7, 8]. A home monitoring programme integrated with home spirometry improved general wellbeing of patients with ILD, and provided opportunities to tailor medication use [7, 9]. Interestingly, oxygen saturation was the most desired parameter to measure at home. This may have been influenced by the COVID-19 pandemic [10], though it is also considered an important measure to monitor disease course and guide titration of oxygen supplementation in ILD, regardless of COVID-19 [2]. Notably, three-quarters of HCPs would like to monitor physical activity levels, which is done infrequently in current practice. Until now, few studies have evaluated the use of physical activity trackers in ILD [11-13]. Physical activity trackers may be useful to monitor and predict disease deterioration over time, encourage patients to exercise, and enable tele-rehabilitation [11-13].

Few HCPs currently use home monitoring applications for research or clinical care, which may be due to the barriers identified in this survey. Most HCPs have no staff available to instruct patients and follow up results, and reimbursement for eHealth is lacking. Technical and analytic issues were encountered in previous home spirometry studies $[14,15]$. This is consistent with our survey results, with technical issues being the most frequently reported hurdle. To facilitate implementation of eHealth in regular care, focus should be on improving technical infrastructure, optimisation of automated alert settings and related legal requirements, establishing financial reimbursement plans, and developing training programmes for patients and caregivers. Attention should be paid to the impact on quality of care and suitability for different patient groups. For research and registry purposes, consensus documents on technical and analytical requirements are needed to ensure standardised collection and privacy compliant sharing. To enable wide-scale implementation of eHealth for patients with ILD both during and after the COVID-19 pandemic, these challenges should be addressed. 
A limitation of the study is the possibility that HCPs with pre-existing interest in eHealth preferentially responded to the survey, leading to selection bias. However, due to the high number of responses from HCPs around the world, we believe our results represent the experiences and opinions on eHealth of a diversity of HCPs.

In conclusion, this study shows that HCPs worldwide are positive towards implementing eHealth applications. These results are encouraging and can be used to shape further integration of eHealth in care and research for patients with ILD.

\section{Gizal Nakshbandi ${ }^{1}$, Catharina C. Moor ${ }^{1}$, Kerri A. Johannson $\oplus^{2}$, Toby M. Maher ${ }^{3}$, Michael Kreuter $\oplus^{4}$ and Marlies S. Wijsenbeek ${ }^{1}$}

${ }^{1}$ Dept of Respiratory Medicine, Erasmus University Medical Centre, Rotterdam, The Netherlands. ${ }^{2}$ Dept of Medicine, University of Calgary, Calgary, Canada. ${ }^{3}$ Keck School of Medicine of University of Southern California, Los Angeles, CA, USA. ${ }^{4}$ Center for Interstitial and Rare Lung Diseases, Thoraxklinik, University of Heidelberg, Germany and German Center for Lung Research, Heidelberg, Germany.

Corresponding author: Marlies Wijsenbeek (m.wijsenbeek-lourens@erasmusmc.nl)

Conflict of interest: G. Nakshbandi has nothing to disclose. C.C. Moor reports grants and other support, paid to her institution, from Boehringer Ingelheim, outside the submitted work. K.A. Johannson reports advisory board and consulting fees, speaker honoraria, travel fees, and a research grant from Boehringer Ingelheim; advisory board fees speaker honoraria from Hoffman La Roche Ltd; advisory board and consulting fees from Theravance and Blade Therapeutics; grants from the Chest Foundation, the University of Calgary School of Medicine and the Pulmonary Fibrosis Society of Calgary; consulting fees and a research grant from the Three Lakes Foundation; and consulting fees from Pliant Therapeutic, all outside the submitted work. T.M. Maher has, via his institution, received industry-academic funding from AstraZeneca and GlaxoSmithKline R\&D, and has received consultancy or speaker's fees from AstraZeneca, Bayer, Blade Therapeutics, Boehringer Ingelheim, Bristol-Myers Squibb, Galapagos, Galecto, GlaxoSmithKline R\&D, IQVIA, Pliant, Respivant, Roche and Theravance. M. Kreuter reports grants and personal fees from Boehringer Ingelheim and Roche, and personal fees from Galapagos, outside the submitted work. M.S. Wijsenbeek reports grants and other support from Boehringer Ingelheim and Hoffman la Roche, and support from Galapagos, Respivant, Galecto, BMS, Novartis, Puretech and NeRRe Therapeutics, outside the submitted work; all grants and fees were paid to her institution.

\section{References}

$1 \quad$ Wijsenbeek M, Cottin V. Spectrum of fibrotic lung diseases. N Engl J Med 2020; 383: 958-968.

2 Raghu G, Collard HR, Egan JJ, et al. An official ATS/ERS/JRS/ALAT statement: idiopathic pulmonary fibrosis: evidence-based guidelines for diagnosis and management. Am J Respir Crit Care Med 2011; 183: 788-824.

3 Antoniou KM, Raghu G, Tzilas V, et al. Management of patients with interstitial lung disease in the midst of the COVID-19 pandemic. Respiration 2020; 99: 625-627.

4 Wong AW, Fidler L, Marcoux V, et al. Practical considerations for the diagnosis and treatment of fibrotic interstitial lung disease during the coronavirus disease 2019 pandemic. Chest 2020; 158: 1069-1078.

5 Drake TM, Docherty AB, Harrison EM, et al. Outcome of hospitalization for COVID-19 in patients with interstitial lung disease. An international multicenter study. Am J Respir Crit Care Med 2020; 202: 1656-1665.

6 Nakshbandi G, Moor CC, Wijsenbeek MS. Home monitoring for patients with ILD and the COVID-19 pandemic. Lancet Respir Med 2020; 8: 1172-1174.

7 Moor CC, Mostard RLM, Grutters JC, et al. Home monitoring in patients with idiopathic pulmonary fibrosis. A randomized controlled trial. Am J Respir Crit Care Med 2020; 202: 393-401.

8 Moor CC, van Leuven SI, Wijsenbeek MS, et al. Feasibility of online home spirometry in systemic sclerosis-associated interstitial lung disease: a pilot study. Rheumatology 2020; 60: 2467-2471.

9 Johannson KA. Remote monitoring in idiopathic pulmonary fibrosis: home is where the bluetooth-enabled spirometer is. Am J Respir Crit Care Med 2020; 202: 316-317.

10 Carroll O, MacCann R, Reilly A, et al. Remote monitoring of oxygen saturation in individuals with COVID-19 pneumonia. Eur Respir J 2020; 56: 2001492.

11 Bahmer T, Kirsten AM, Waschki B, et al. Clinical correlates of reduced physical activity in idiopathic pulmonary fibrosis. Respiration 2016; 91: 497-502. 
12 Moor CC, van den Berg CAL, Visser LS, et al. Diurnal variation in forced vital capacity in patients with fibrotic interstitial lung disease using home spirometry. ERJ Open Res 2020; 6: 00054-02020.

13 Drent M, Elfferich M, Breedveld E, et al. Benefit of wearing an activity tracker in sarcoidosis. J Pers Med 2020; 10: 97.

14 Maher TM, Corte TJ, Fischer A, et al. Pirfenidone in patients with unclassifiable progressive fibrosing interstitial lung disease: a double-blind, randomised, placebo-controlled, phase 2 trial. Lancet Respir Med 2020; 8: 147-157.

15 Noth I, Cottin V, Chaudhuri N, et al. Home spirometry in patients with idiopathic pulmonary fibrosis: data from the INMARK trial. Eur Respir J 2021; 58: 2001518. 